Noname manuscript No.

(will be inserted by the editor)

\title{
Dynamic Considerations of Heel-Strike Impact in Human Gait
}

\author{
Javier Ros • Josep M. Font-Llagunes • \\ Aitor Plaza • József Kövecses
}

Received: date / Accepted: date

\begin{abstract}
Based on the impulsive-dynamics formulation, this article presents the analysis of different strategies to regulate the energy dissipation at the heel-strike event in the context of human locomotion. For this purpose, a seven-link $2 D$ human-like multibody model based on anthropometric data is used. The model captures the most relevant dynamic and energetic aspects of the heel-strike event in the sagittal plane. The pre-impact mechanical state of the system, around which the analysis of the heel impact contribution to energy dissipation is performed, is defined based on published data. In the context of the proposed impulsivedynamics framework, different realistic strategies that the subject can apply to modify the impact dynamics are proposed and analyzed, namely, the trailing ankle push-off, the torso configuration and the degree of joint blocking in the colliding leg. Detailed numerical analysis and discussions are presented to quantify the ef-
\end{abstract}

J. Ros

Department of Mechanical Engineering

Public University of Navarre

Campus de Arrosadia, 31006 Pamplona, Navarre, Spain

Tel.: + 34-948166215

Fax: +34-948169099

E-mail: jros@unavarra.es

J. M. Font-Llagunes

Department of Mechanical Engineering, and Biomedical Engineering Research Centre.

Universitat Politècnica de Catalunya

Diagonal 647, 08028 Barcelona, Catalonia, Spain

E-mail: josep.m.font@upc.edu

A. Plaza

Department of Mechanical Engineering

Public University of Navarre

Campus de Arrosadia, 31006 Pamplona, Navarre, Spain

E-mail: aitor.plaza@unavarra.es

J. Kövecses

Department of Mechanical Engineering, and Centre for Intelligent Machines

McGill University

817 Sherbrooke St. West, H3A 2K6 Montreal, Quebec, Canada

E-mail: jozsef.kovecses@mcgill.ca

This is a post-peer-review, pre-copyedit version of an article published in Multibody System Dynamics.

The final authenticated version is available online at: https://doi.org/10.1007/s11044-015-9460-0 
fects of the mentioned strategies.

Keywords: Biomechanics, Bipedal walking, Impact dynamics, Energy analysis, Human gait.

\section{Introduction}

The dynamics of human locomotion is a major subject in the area of biomechanical engineering. The research in that field is very important for different purposes, e.g., to improve the performance of athletes in sports, to design rehabilitation or prosthetic devices to assist disabled subjects or to develop better bipedal walking robots based on the physical principles of human locomotion.

The human gait cycle is composed of two phases: the stance phase, which lasts approximately the $60 \%$ of the cycle, and the swing phase, which lasts the other $40 \%$. It was observed that during human gait, the motion of the stance leg is similar to that of an inverted pendulum rotating about the ankle and the swing leg also performs a pendulum-like motion rotating about the hip joint [1], [2]. During this phase the gait progression can be considered assisted by four foot rockers: heel rocker, ankle rocker, forefoot rocker and toe rocker [3]. The motion of the swing leg is mainly passive in nature and it requires little muscle activity to be performed [4]. Conversely, the motion of the stance leg does require an energy input to restore the losses that occur at heel-strike impact (at the end of the swing phase). Based on these assumptions, simple models that provided useful information regarding the dynamics and energetic aspects of human (bipedal) locomotion were developed $[5]$.

Heel strike is known to be a major contributor to the stability of bipedal locomotion [6], [7], [8], [9]. The change of motion that the system experiences at each heel strike has a strong stabilizing effect. For example, the trajectory of the centre of mass (which is approximately placed at the hip joint height) is redirected from one pendular arc to the next in walking motion [10], [11], or from one parabolic free-flight arc to the next in running motion [12]. On the other hand, from a dynamical point of view, the energy dissipating nature of the heel impact can also stabilize the movement. The associated phase-space volume contraction has been shown to be a necessary condition for stability [13].

Passive dynamic walking refers to simple mechanical models that are able to walk down a slightly inclined walkway with no external actuation [14], [15]. The work on this type of walkers showed that it was possible to obtain orbitally stable limit cycles, with remarkably human-like motion, without any kind of actuation and control. It is worth noting that the analysis of passive dynamic walking has led to important insights regarding the basic principles of human locomotion. For example, in [16] and [10] a simple passive model (the so-called simplest walking model) was used to analyze the energetic cost of human walking. In those papers, useful conclusions were drawn via combining experimental data with the mechanics associated with this simple biomechanical model. The model was also used in $[12]$ to find energetically optimal walking and running gait patterns by means of computer optimization.

It is known that for a given passive walker, there is a slope limit after which it is impossible to obtain stable limit cycles. However, this limit can be exceeded if 
extra dissipation is introduced in the system. For example, in [13] damping at the hip joint was used for this purpose. This supports the idea that human locomotion is partially controlled by regulating the amount of energy that is dissipated at heel impact. It was shown in [17], that the mass distribution of the system, and the torso and stance leg angles at heel strike have an influence on the energy loss.

The high impact forces occurring at heel landing can initiate soft-tissue vibrations that contribute significantly to energy dissipation [18]. In this sense, it is interesting to highlight the work by Boyer and Nigg [19], [20], which introduced the idea of muscle tuning before impact. The authors showed that the activation of leg muscles before heel landing was correlated with the vibration of the softtissue compartment and, therefore, to the energy dissipation. Also, it was shown in [21] that the knee joint stiffness influences the magnitude of impact forces. Thus, muscle activation before and during impact is used, not only to control the joint compliances and the leg configuration at impact, but also to control the amount of energy loss.

It is important to note that the mentioned energy dissipating mechanisms at heel impact, although active in nature, have a very low energetic cost. Muscles do not directly remove the dissipated energy, they rather alter the stiffness and dissipating capabilities at a given joint, and thus they indirectly produce the actual energetic dissipation. For example, during the impact a portion of kinetic energy is transferred from the rigid body motion to the soft tissue in the form of vibration. This energy gets dissipated as the soft-tissue vibrations damp out.

As we will see the amount of energy that can be dissipated in this way can be controlled through the rigidization of different joints. These joint blockings act like a set of switches that control the flow of energy between the different parts of the body and to the soft-tissue vibrations. Therefore, the energy needed to activate these switches is much smaller than the one that they control. This implies that the heel impact event can be used as one of the most efficient ways to regulate mechanical energy dissipation from a biomechanical point of view. Other forms of energy removal will require work done by the leg muscles during the stance phase.

Due to the relevant role of heel strike in the dynamics of walking, in this work, we are interested in the analysis of the dissipating capabilities of heel impact using different strategies.

The impulsive framework is not able to give a detailed description of the forces and velocities during the usually short impulsive event, but it is able to predict exactly the after-impact velocity and the constraint impulses ${ }^{1}$. This is enough for the determination of the heel strike dissipating capabilities. The only requirement is that the characteristic time of the impulsive event is small enough so that changes in position are not appreciable. This requirement has been found to be satisfied with sufficient accuracy for the heel strike event. For instance, this can be observed in the experiments in [22].

An important advantage of the impulsive framework is that just inertial properties are required; stiffnesses and damping properties are not needed. This simplifies enormously the model setup. Moreover, in closely related studies, [25, 26], it was shown that a more detailed compliant contact model yields very similar results to those obtained with the impulsive framework.

\footnotetext{
1 time integral of the constraint forces during the impact
} 
A seven-link $2 D$ human-like multibody model is chosen to simulate the effect of different actuation mechanisms on the heel strike event. Inertial and geometric properties are based on the anthropometric data in [27]. This model captures the most relevant contributions to the impulsive dynamics of human walking in the sagittal plane.

Based on bibliographic data for human walking [22], we define a pre-impact reference configuration and velocity, and perform an analysis of the heel impact contribution to dissipation in that state neighborhood. We will consider different realistic strategies that the subject can apply to regulate energy dissipation at impact: pre-impact velocities, pre-impact torso configuration, and total or partial blocking of the leading leg joints.

The symbolic multibody program 3D_MEC [28] was used as a pre-processor, to setup the dynamic model and kinematic set of equations, and as a post-processor to present some of the results. The actual numerical computations were done using MATLAB, employing the exporting capabilities of 3D_MEC.

\section{Biomechanical Model of the Human Body}

In this section, the multibody model used to study the dynamics of human locomotion at the heel-strike event using the impulsive framework is presented. First, the model, that is, the kinematic parametrization and the geometric and inertial parameters employed are described. Second, the configuration at impact and the method proposed to determine meaningful pre-impact velocities are presented and justified. Finally, the equations of the impulsive dynamics formalism and the postimpact kinematic constraints used in this study are described. We also explain the way in which we introduce the representation for the partial blocking of joints within the proposed dynamics formalism.

\subsection{Model Parametrization}

The model is shown in Fig 1, and is composed of seven segments: the TorsoPelvis segment, the two Thighs, the two Shanks and the two Feet. These links are coupled by the Hip, Knee and Ankle joints, which are all assumed to be ideal revolute joints. As shown in Fig. 2 the Torso-Pelvis configuration is given with absolute coordinates $x, y$ of the Lumbar Joint Center $(L J C)$ and absolute rotation $\theta$. Relative coordinates $\theta_{H}^{R}, \theta_{K}^{R}$ and $\theta_{A}^{R}$ are used to position the right thigh, leg and foot, with respect to the preceding element. Analogously $\theta_{H}^{L}, \theta_{K}^{L}$ and $\theta_{A}^{L}$ are used for the segments in the left side of the body. We have $n=9$ generalized coordinates that can be represented with the array

$$
\mathbf{q}=\left[x, y, \theta, \theta_{H}^{R}, \theta_{K}^{R}, \theta_{A}^{R}, \theta_{H}^{L}, \theta_{K}^{L}, \theta_{A}^{L}\right]^{T}
$$

The kinematic an dynamic parameters of the model are obtained from the anthropometric, geometric and inertial, data for normal subjects given in [27]. These data are given in terms of the total body mass that is set to $75 \mathrm{~kg}$. 


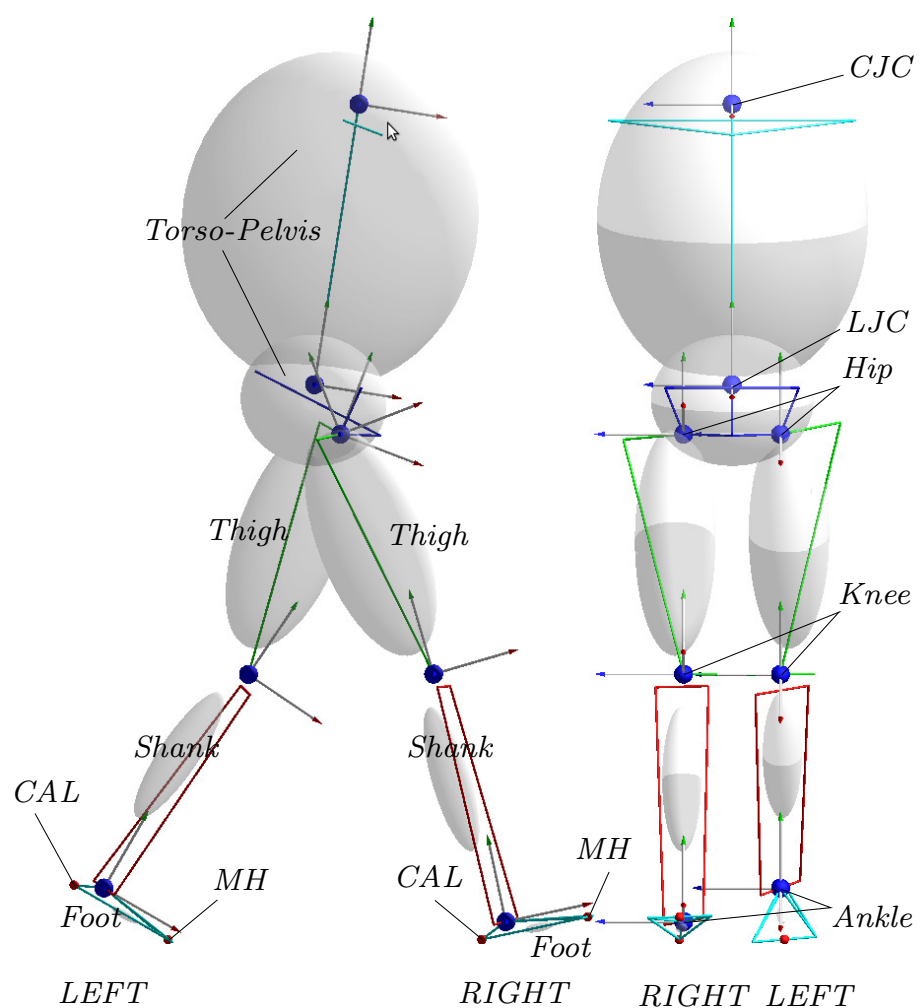

Fig. 1 Biomechanical multibody model at the pre-impact configuration.

The kinetic energy, $T=T(\mathbf{q}, \dot{\mathbf{q}})$, of the system is symbolically obtained and expressed as,

$$
T=\frac{1}{2} \dot{\mathbf{q}}^{T} \mathbf{M} \dot{\mathbf{q}}
$$

where $\mathbf{M}=\mathbf{M}(\mathbf{q})$ is the $n \times n$ mass matrix.

\subsection{Pre-impact Configuration and Velocity}

The configuration depicted in Fig. 1 is the reference pre-impact configuration used in this study. It is based on the data of [22] and adjusted for normal walking condition and for a subject with average height.

For the simulations, impact is assumed to take place at the calcaneus marker $(C A L)$ of the colliding (or leading) foot. This belongs to the right leg, which is the swing leg before the impact. The left foot is assumed to contact the ground at the mid point between the 1st and 5th metatarsal head markers $(M H)$. The left (or trailing) leg is the stance leg before the impact.

A procedure, based on a set of kinematic assumptions that should be fulfilled at the pre-impact instant, was devised to obtain velocity data coherent with experimental observations of normal walking [22]. The following kinematic assumptions were used to define the velocities of the system at the pre-impact time: 
- The Torso-Pelvis segment does not rotate with respect to the right Thigh, $\dot{\theta}_{H}^{R}=0$, and the horizontal velocity component of the $L J C$ is defined based on the average horizontal velocity, $v_{0}=1.61 \mathrm{~m} / \mathrm{s}$, for that point estimated based on [22]. The kinematic equation can thus be expressed as $\mathbf{v}(L J C) \cdot \mathbf{e}_{x}-v_{0}=0$. Where $\mathbf{v}(L J C)$ represents the velocity of the $L J C$ and $\mathbf{e}_{x}$ is the unit vector in the $x$ direction.

- The $C A L$ point of the colliding foot has zero horizontal (tangential) velocity to avoid sliding at foot landing. Then the kinematic equation reads $\mathbf{v}\left(C A L^{R}\right) \cdot \mathbf{e}_{x}=$ 0 .

- The knee and ankle joints of the colliding leg have zero relative angular velocities before the impact, $\dot{\theta}_{K}^{R}=0$ and $\dot{\theta}_{A}^{R}=0$.

- The $M H$ point of the left foot has zero pre-impact velocity, $\mathbf{v}(M H)=\mathbf{0}$, and the knee joint of that leg has zero relative angular velocity, $\dot{\theta}_{K}^{L}=0$. The relative angular velocity of the left ankle is left as a variable that allows for experimenting with different pre-impact landing velocities $\dot{\theta}_{A}^{L}=\omega_{0}$. 
Thus, the pre-impact generalized velocities, $\dot{\mathbf{q}}^{-}$, can be obtained as the solution of the following linear system of equations:

$$
\left[\begin{array}{c}
\dot{\theta}_{H}^{R} \\
\mathbf{v}(L J C) \cdot \mathbf{e}_{x}-v_{0} \\
\mathbf{v}\left(C A L^{R}\right) \cdot \mathbf{e}_{x} \\
\dot{\theta}_{K}^{R} \\
\dot{\theta}_{A}^{R} \\
\mathbf{v}(M H) \cdot \mathbf{e}_{x} \\
\mathbf{v}(M H) \cdot \mathbf{e}_{y} \\
\dot{\theta}_{K}^{L} \\
\dot{\theta}_{A}^{L}-\omega_{0}
\end{array}\right]=[\mathbf{0}]
$$

The above assumptions can be justified, for example, based on the experimental work reported in [22]; see Part II, pp. 345-353, specially Figs. 5 and 6. The angles in this reference are defined as here. Angles just before $100 \%$ completion of the gait cycle (pre-impact) justify an almost zero angular velocity for leading Pelvis, Hip, Knee and Ankle at the pre-impact configuration. In those figures, the impulsive nature of the heel strike event is apparent and related to the sudden slope change at the transition between $100 \%$ and $0 \%$. At about $50 \%$ of the cycle we can find the values for the trailing leg angles, the trailing knee angle is close to a minimum so its derivative is near ${ }^{2}$ zero. The pushing of the trailing ankle can be observed at about $30-50 \%$ of the cycle. The associated angular velocity, $\dot{\theta}_{A n}^{L}$., which is associated with the rotation of the ankle joint, will be used as an additional degree of freedom (DoF) to negotiate the initial pre-impact landing velocity.

\subsection{Impulsive Dynamic Equations and Constraints}

Assuming that the applied forces acting on the system are not impulsive, the dynamics of the impulsive motion phase can be characterized by impulse-momentum level dynamic equations [23, 24]:

$$
\mathbf{M}\left(\dot{\mathbf{q}}^{+}-\dot{\mathbf{q}}^{-}\right)=\mathbf{A}^{T} \bar{\lambda}
$$

and the following kinematic constraints at the post-impact instant

$$
\mathbf{A} \dot{\mathbf{q}}^{+}=\mathbf{b}^{+}
$$

$\mathbf{M}$ is the $n \times n$ mass matrix for the parametrization $\mathbf{q}$, and $\mathbf{A}=\mathbf{A}(\mathbf{q})$ is the $m \times n$ Jacobian of the kinematic constraints enforced at $t^{+}$. The superscripts "_" and "+" denote the pre- and post-impact instants. Vector $\mathbf{A}^{T} \overline{\boldsymbol{\lambda}}$ is the generalized impulse associated to the constraint forces. We introduce $\mathbf{b}^{+}$in the constraint equations for generality, so we can consider both rheonomic or scleronomic impulsive constraints ${ }^{3}$. These equations allow to solve for $\dot{\mathbf{q}}^{+}$and $\bar{\lambda}$ given the initial velocity $\dot{\mathbf{q}}^{-}$.

For the problem at hand, the scleronomic constraints are defined as follows:

\footnotetext{
2 The sensitivity to the trailing leg velocities found at the impulsive event is very small.
}

3 For scleronomic constraints, $\mathbf{b}^{+}=\mathbf{0}$. 
- The post-impact contact and non-sliding conditions at the right $C A L$,

$$
\begin{aligned}
& \mathbf{v}\left(C A L^{R}\right) \cdot \mathbf{e}_{x}=0 \longrightarrow \text { Leading Foot } C A L \text { non-sliding } \\
& \mathbf{v}\left(C A L^{R}\right) \cdot \mathbf{e}_{y}=0 \longrightarrow \text { Leading Foot } C A L \text { contact }
\end{aligned}
$$

That is, the colliding point must stay in contact with the ground and not slide with respect to the ground after the impact. This is a reasonable and widely used assumption in the analysis of human locomotion.

- The post-impact contact and non-sliding conditions at the trailing $M H$,

$$
\begin{aligned}
& \mathbf{v}\left(M H^{L}\right) \cdot \mathbf{e}_{x}=0 \longrightarrow \text { Trailing Foot } M H \text { non-sliding } \\
& \mathbf{v}\left(M H^{L}\right) \cdot \mathbf{e}_{y} \geq 0 \longrightarrow \text { Trailing Foot } M H \text { unilateral contact, }
\end{aligned}
$$

For a normal gait, it is experimentally observed that $\mathbf{v}\left(M H^{L}\right)=\mathbf{0}$ after the impact. Nevertheless, we consider "a priori" the possibility of contact loss $\mathbf{v}\left(M H^{L}\right) \cdot \mathbf{e}_{y} \geq 0$ to check the dynamic consistency of our model ${ }^{4}$.

In this paper we consider partial blocking of a given set of joints. We consider that muscles involved in the motion of a particular joint can be strongly stressed during the impact so that they can block the relative motion of the given joint to some extent.

Within the impulsive framework adopted, such a partial blocking implies actions of impulsive nature. For example, it was shown in [21] that the knee joint stiffness influences the magnitude of impact forces, also in [18] it has been demonstrated that the impact at heel landing initiates soft-tissue vibrations that contribute to energy dissipation. As it has been commented in the introduction, muscular action can be used to tune the stiffness and impact forces. Also, in normal walking the leading leg knee is stiffened during the heel-strike phase, avoiding the flexion motion of this joint to some extent, making the thigh and shank to behave similar to a single rigid body. Obviously, the leading leg ankle stiffness can be altered through a similar mechanism.

Thus, we can say that muscle activation before and during impact is used, not only to control the joint compliances and the leg configuration at impact, but also to control the amount of energy loss during the impact. Within the impulsive framework employed, these actuation mechanisms need to be addressed using impulsive joint actions. We propose to deal with the so-called partial blocking kinematically, by specifying the post-impact relative velocities for the set of joints that are participating in the partial blocking. In other words, introducing rheonomic impulsive constraints for the different joints of the model to account for partial joint blocking and the related dissipation phenomena:

- The different blocking strategies can be studied taking different rheonomic constraint combinations from the following set,

$$
\begin{aligned}
\dot{\theta}_{A}^{R}-\dot{\theta}_{A}^{R+} & =0 \longrightarrow \text { Leading Ankle partial blocking } \\
\dot{\theta}_{K}^{R}-\dot{\theta}_{K}^{R+} & =0 \longrightarrow \text { Leading Knee partial blocking } \\
\dot{\theta}_{H}^{R}-\dot{\theta}_{H}^{R+} & =0 \longrightarrow \text { Leading Hip partial blocking }
\end{aligned}
$$

4 The presence of this unilateral constraint implies a "complementarity problem". We deal with this via looking for two different solutions, one "with" and the other "without" this constraint enforced. Then, we choose the physically meaningful one for which $\mathbf{v}\left(M H^{L}\right) \cdot \mathbf{e}_{y}=$ 0 and $\bar{\lambda}>0$ or $\mathbf{v}\left(M H^{L}\right) \cdot \mathbf{e}_{y}>0$ and $\bar{\lambda}=0$ must be satisfied. 
Where $\dot{\theta}_{j}^{R+}$ is set to zero to impose total blocking of joint $j=(A, K, H)$.

\section{Decomposition of the Impulsive Motion Equations and Kinetic Energy}

Based on the constraint Jacobian in Eq. (4), the tangent space of the configuration manifold of the walking system can be decomposed to the spaces of constrained and admissible motions (SCM and SAM), respectively, for the pre-impact and post-impact instants [29], [30]. This will then also hold for the entire duration of the contact onset, since the configuration of the system is assumed constant during this short period of time.

The two subspaces can be defined so that they are orthogonal to each other with respect to the mass metric of the tangent space of the freed system (constraints in Eq. (4) are relaxed). This decomposition can be accomplished via two $n \times n$ dimensional projector operators [29], [30]. Assuming that the impulsive constraints in Eq. (4) are independent of each other, the projector associated with the SCM can be written as

$$
\mathbf{P}_{c}=\mathbf{M}^{-1} \mathbf{A}^{T}\left(\mathbf{A} \mathbf{M}^{-1} \mathbf{A}^{T}\right)^{-1} \mathbf{A},
$$

and the projector for the SAM can be obtained as

$$
\mathbf{P}_{a}=\mathbf{I}-\mathbf{P}_{c}=\mathbf{I}-\mathbf{M}^{-1} \mathbf{A}^{T}\left(\mathbf{A} \mathbf{M}^{-1} \mathbf{A}^{T}\right)^{-1} \mathbf{A}
$$

where $\mathbf{I}$ denotes the $n \times n$-dimensional identity matrix. These projectors are not symmetric, which is a direct consequence of the nature of the metric of the tangent space. Based on them, the generalized velocities of the system can be decomposed as

$$
\dot{\mathbf{q}}=\mathbf{P}_{c} \dot{\mathbf{q}}+\mathbf{P}_{a} \dot{\mathbf{q}} \equiv \mathbf{v}_{c}+\mathbf{v}_{a},
$$

which represent the two components associated with the subspaces. It is interesting to note that in general $\mathbf{v}_{c}=\mathbf{P}_{c} \dot{\mathbf{q}}$ and $\mathbf{v}_{a}=\mathbf{P}_{a} \dot{\mathbf{q}}$ are non-holonomic quantities. Generalized impulses can also be decomposed using the transpose of the operators given above [29]. Then, for the impulsive case we have

$$
\overline{\mathbf{f}}=\mathbf{P}_{c}^{T} \overline{\mathbf{f}}+\mathbf{P}_{a}^{T} \overline{\mathbf{f}} \equiv \overline{\mathbf{f}}_{c}+\overline{\mathbf{f}}_{a}
$$

Based on Eqs. (2) and (15), it can be shown that the kinetic energy can also be decomposed to two independent parts as

$$
T=\frac{1}{2} \mathbf{v}_{c}^{T} \mathbf{M} \mathbf{v}_{c}+\frac{1}{2} \mathbf{v}_{a}^{T} \mathbf{M} \mathbf{v}_{a} \equiv T_{c}+T_{a}
$$

which is a consequence of the projectors in (13) and (14) being orthogonal with respect to the system mass matrix, i.e., $\mathbf{P}_{c}^{T} \mathbf{M} \mathbf{P}_{a}=\mathbf{P}_{a}^{T} \mathbf{M} \mathbf{P}_{c}=\mathbf{0}$. Therefore, using Eq. (15) in Eq. (2), $\mathbf{v}_{c}^{T} \mathbf{M} \mathbf{v}_{a}=\mathbf{v}_{a}^{T} \mathbf{M} \mathbf{v}_{c}=0$. Any impulse arising in the space of constrained motion will change only $T_{c}$, leaving $T_{a}$ unaffected and vice versa [32]. That is, the enforcing of the constraints given by Eq. (5) gives rise to impulses which will directly influence quantities in the space of constrained motion only. 
Based on the above, it can be shown that the impulse-momentum level dynamic equations in Eq. (4) can be decoupled to

$$
\mathbf{M}\left(\mathbf{v}_{c}^{+}-\mathbf{v}_{c}^{-}\right)=\mathbf{A}^{T} \bar{\lambda}
$$

which are the impulse-momentum level dynamic equations associated with the SCM, and

$$
\mathbf{M}\left(\mathbf{v}_{a}^{+}-\mathbf{v}_{a}^{-}\right)=\mathbf{0}
$$

which describe the impulsive dynamics associated with the SAM. From Eq. (19) and using that $\mathbf{M}$ is positive definite, it is immediately visible that $\mathbf{v}_{a}^{+}=\mathbf{v}_{a}^{-}$. Based on Eqs. (4) and (15) it can also be concluded that $\mathbf{v}_{c}^{+}={ }^{g} \mathbf{P}_{c}^{l} \mathbf{b}^{+}$, where ${ }^{g} \mathbf{P}_{c}^{l}$ is the $n \times m$-dimensional matrix that projects SCM's components in local parametrization to components in global parametrization[29]:

$$
{ }^{g} \mathbf{P}_{c}^{l}=\mathbf{M}^{-1} \mathbf{A}^{T}\left(\mathbf{A} \mathbf{M}^{-1} \mathbf{A}^{T}\right)^{-1} .
$$

Then, using the above results the following expression to solve for the post-impact generalized velocities $\dot{\mathbf{q}}^{+}$is obtained

$$
\dot{\mathbf{q}}^{+}=\mathbf{v}_{a}^{-}+{ }^{g} \mathbf{P}_{c}^{l} \mathbf{b}^{+}=\mathbf{P}_{a} \dot{\mathbf{q}}^{-}+{ }^{g} \mathbf{P}_{c}^{l} \mathbf{b}^{+} .
$$

Based on Eqs. (4), (15) and (18) we can also obtain the solution for the generalized constraint impulses as

$$
\bar{\lambda}=\left(\mathbf{A} \mathbf{M}^{-1} \mathbf{A}^{T}\right)^{-1}\left(\mathbf{b}^{+}-\mathbf{A} \dot{\mathbf{q}}^{-}\right)
$$

that make the velocity of the colliding foot zero and accomplish the total or partial blocking of joints. The last expression clearly shows that $\mathbf{b}^{+}$can be used to control the impulses $\bar{\lambda}$ and vice versa.

We define the following non-dimensional energetic performance index, $\xi$, to quantify the energetic aspects of heel impact:

$$
\xi=\frac{T^{-}-T^{+}}{T^{-}}=\frac{T_{c}^{-}-T_{c}^{+}}{T^{-}},
$$

where $\xi$ represents the ratio of the kinetic energy lost at heel strike to the preimpact kinetic energy, i.e., the local energetic efficiency of the impact [31].

It is easy to see that,

$$
\begin{aligned}
T_{c}^{+} & =\frac{1}{2}\left(\mathbf{b}^{+}\right)^{T}{ }^{g} \mathbf{P}_{c}^{l T} \mathbf{M}{ }^{g} \mathbf{P}_{c}^{l} \mathbf{b}^{+}=\frac{1}{2}\left(\mathbf{b}^{+}\right)^{T} \mathbf{W}_{c} \mathbf{b}^{+} \\
T_{c}^{-} & =\frac{1}{2}\left(\mathbf{b}^{-}\right)^{T}{ }^{g} \mathbf{P}_{c}^{l^{T}} \mathbf{M}{ }^{g} \mathbf{P}_{c}^{l} \mathbf{b}^{-}=\frac{1}{2}\left(\mathbf{b}^{-}\right)^{T} \mathbf{W}_{c} \mathbf{b}^{-}
\end{aligned}
$$

where $\mathbf{b}^{-}=\mathbf{A} \dot{\mathbf{q}}^{-}$, and $\mathbf{W}_{c}$ is the mass matrix associated to the local parametrization in the SCM.

Based on Eqs. (15) and (18), and having in mind that $\mathbf{v}_{a}^{T} \mathbf{M} \mathbf{v}_{c}=0$, it is also possible to see that

$T_{c}^{+}-T_{c}^{-}=\frac{1}{2}\left(\mathbf{v}_{c}^{+}+\mathbf{v}_{c}^{-}\right)^{T} \mathbf{M}\left(\mathbf{v}_{c}^{+}-\mathbf{v}_{c}^{-}\right)=\frac{1}{2}\left(\mathbf{b}^{+}+\mathbf{b}^{-}\right)^{T} \bar{\lambda}=\frac{1}{2}\left(\mathbf{b}^{+}+\mathbf{b}^{-}\right)^{T} \mathbf{W}_{c}\left(\mathbf{b}^{+}-\mathbf{b}^{-}\right)$.

This gives an explicit relationship between the impulses generated by the contact onset, and the kinetic energy that is lost in the contact event. 


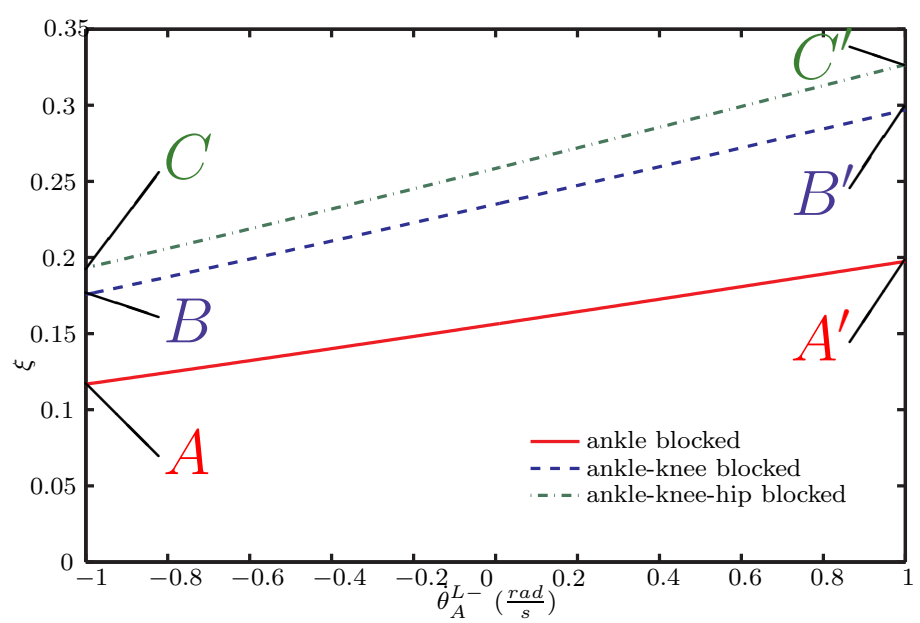

Fig. 3 Simulation Group I: Energetic performance index, $\xi$, vs. pre-impact trailing ankle angular velocity, $\omega_{0}$.

\section{Simulation Results and Discussion}

In this section, the kinetic energy dissipation effectiveness of different regulation strategies at the heel strike event is analyzed and discussed. We present three groups of simulations covering feasible variations of: the pre-impact trailing ankle angular velocity -Section 4.1-, the configuration of the torso -Section 4.2-, and the degree of leading ankle blocking -Section 4.3-, in combination with complete blocking of other joints of the colliding leg.

The gait of a normal subject with pre-impact configuration and velocity determined as described in Section 2.2 is used in these analyses. Also, the decomposition of the kinetic energy at the pre-impact instant, described in Section 3, is used in the discussion.

\subsection{Simulation Group I: Effect of pre-impact trailing ankle angular velocity}

The pre-impact trailing ankle angular velocity is changed while maintaining the horizontal velocity of the hip and the zero horizontal velocity of the colliding heel. To set up this initial condition, the system of equations (3) is solved after setting $\omega_{0}$ to the desired value of trailing ankle pre-impact angular velocity. This change influences the impact velocity of the heel in the normal direction and thus the amount of energy removed at the strike, as was pointed out in [33]. For a real subject, this can be achieved by changing the strength with which the trailing foot ankle pushes upwards immediately before the heel-strike.

Fig. 3 presents the plots for the energetic performance index, $\xi$, defined in Eq. (23), for different values of the pre-impact angular velocity of the trailing ankle, $\dot{\theta}_{A}^{L-}$. Note that, due to the parametrization used, negative values for the angular velocity indicate that the trailing ankle is pushing the body upwards. Simulations with no blocked joints present a very low dissipation $(\approx 2 \%)$ and they 
are not too realistic, so they are not represented. In the figure, the continuous red line presents the results for the case when only the leading ankle is blocked, the blue dashed line for the case when both the leading ankle and knee are blocked, and the dash-point green line for the case when the three joints of the leading leg are blocked after impact.

From these results, the following conclusions can be drawn:

1. If the leading ankle is blocked, the energy dissipated can go up to $20 \%$ for the analyzed push-off range.

2. If the leading knee is blocked additionally, the dissipation can go up to $30 \%$.

3. If the leading hip is blocked additionally, the dissipation increases up to $35 \%$.

4. The variation of the trailing ankle push-off can be used to regulate the energy dissipated at heel strike. For the analyzed range, this mechanism can regulate up to the $50 \%$ of the dissipable energy for the considered set of blocked joints combinations.

The effect of the push-off and of the blocked joints on the energetic performance index, $\xi$, can be explained in terms of the impulsive motion decomposition presented in Section 3. The space of motion defined by the configuration -that is the same for all simulations- can be splited out into the SCM and SAM whose shape and dimensions are dependent on the set of blocked joints. As has been shown, during the impact, all the energy in SCM is dissipated while that in the SAM does not change.

The mentioned low level of dissipation when no joint is blocked is due to the fact that the accommodation of the heel to the after impact zero velocity condition is mainly accomplished with the help of the rotation of the colliding foot ${ }^{5}$. Taking this situation as reference, it is observed that the blocking of the ankle alone greatly increases the dissipation, and that the additional blocking of the knee and hip further increases it. These increments in kinetic energy dissipation are related to the change of shape and dimension of the SCM and SAM introduced by the growing number of blocked joints: the dimension of SAM decreases while the one of the SCM increases accordingly. This gives more room for energy in the SCM, and therefore for dissipation.

For a given set of blocked joints, a greater push-off helps, not only to increase the total pre-impact energy, $T^{-}$, but mainly to lower the amount of energy in the $\mathrm{SCM}, T_{c}$, and, accordingly, to increase the energy in the SAM, $T_{a}$. This is due to the fact that ankle push-off lowers the normal colliding velocity of the heel. This puts the paramount role of trailing ankle push-off in normal walking into a new perspective.

The energetic performance index gives a useful but limited information. Figs. 4 and 5, although in a limited number of points, give additional information that is helpful to understand the heel strike event dynamics. In these figures, the orthogonal decomposition of the dynamics at heel strike at six representative simulation points is presented: In points $A, B$ an $C$ the leading ankle; leading ankle and knee; and leading ankle, knee and hip are blocked, respectively, and there is a maximum upward push-off of the trailing ankle. Points $A^{\prime}, B^{\prime}$ and $C^{\prime}$ present the same joint blocking conditions but, in this case, with the ankle angular velocity $\omega_{0}$ in the

\footnotetext{
5 Note that, after the heel strike event the whole foot will eventually collide with the ground, and then the rotating energy will get mostly dissipated. This second collision mechanism is not analyzed in this work.
} 

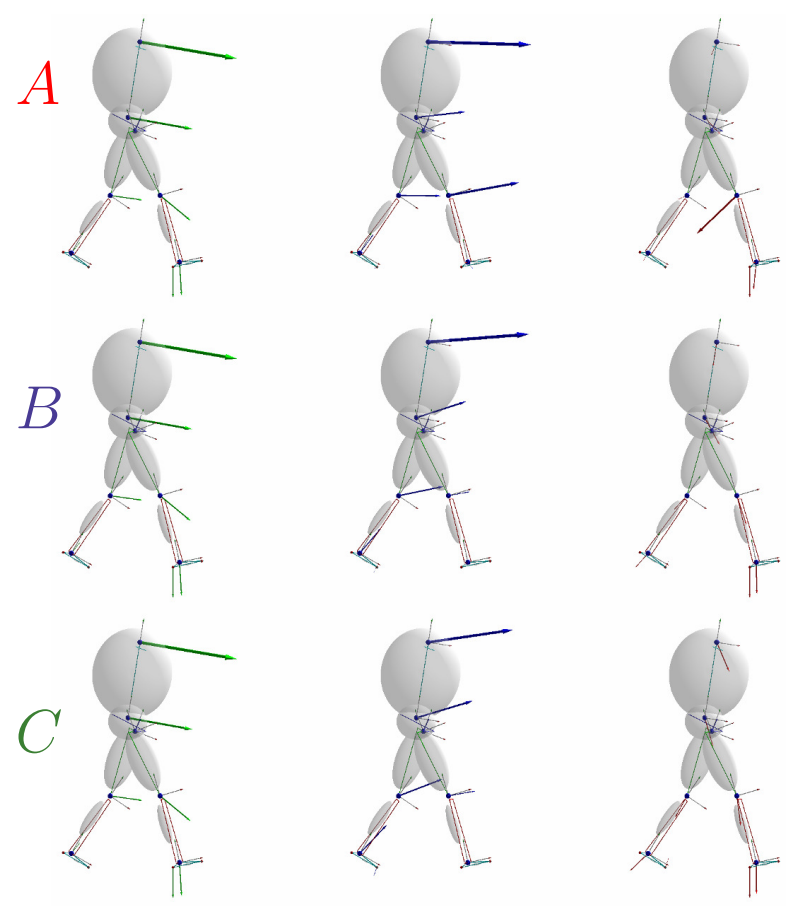

Fig. 4 Mass orthogonal decomposition of the movement for points $A, B$ and $C$. From left to right: total (SM), admissible (SAM) and constrained (SCM) pre-impact velocities of reference points.

opposite end of the analyzed range (actually, in the other direction). From these figures, it can be concluded that the additional blocking of the knee alters not only the magnitude of the different velocities in the SAM and SCM, but also their relative magnitudes. This relative change is not as significant when the leading hip is additionally blocked. This leads us to the conclusion that the additional hip blocking reinforces the dissipation introduced by the knee, with no relevant side effects on the distribution of velocities. These figures also allow us to see that the discussed blocking of the ankle alone produces an important after impact velocity of the leading knee center, that looks not compatible with normal walking gait. Nevertheless, it can be of interest in other situations, for example when impact forces need to be kept small.

\subsection{Simulation Group II: Effect of torso configuration}

In this section, the effect of the pre-impact Torso-Pelvis orientation is analyzed. Varying such orientation allows for modifying the inertia distribution and therefore the energetics of the impact. The influence of this configuration change in the energetic dissipation at heel strike impact has already been shown for a compass-like 
Fig. 5 Mass orthogonal decomposition of the movement for the points $A^{\prime}, B^{\prime}$ and $C^{\prime}$. From left to right: total (SM), admissible (SAM) and constrained (SCM) pre-impact velocities of reference points.

passive walker in [17]. To set up the pre-impact state, the system of equations (3) is solved after setting the Torso-Pelvis angle $\theta$ to the desired value, using a constant value of $\omega_{0}=-1 \mathrm{rad} / \mathrm{s}$ for the trailing ankle push-off. The initial velocity vector $\dot{\mathbf{q}}^{-}$is coincident with that used for simulation points $A, B$ and $C$ exhibiting minimum energy dissipation in Simulation Group $I$, but varying the torso angle.

Fig. 6 presents the plots for the energetic performance index, $\xi$, for different values of the Torso-Pelvis angle deviation, $\Delta \theta=\theta-\theta_{n}$, where $\theta_{n}$ is the absolute torso angle at the reference heel-strike position obtained from [22]). Due to the parametrization used, negative values of $\theta$ indicate that the torso is leaning forward, according to Fig. 2. As before, three cases are considered in the Fig. 6: leading ankle blocked; ankle and knee blocked; and ankle, knee and hip blocked.

We summarize in the following points the main conclusions obtained from this analysis:

1. If the leading ankle is blocked, the energy dissipated can be greater than $13 \%$. In this situation, the orientation of the Torso-Pelvis can be used to modestly regulate the energy dissipated at the heel-strike down to a $10 \%$ for the range analyzed. 


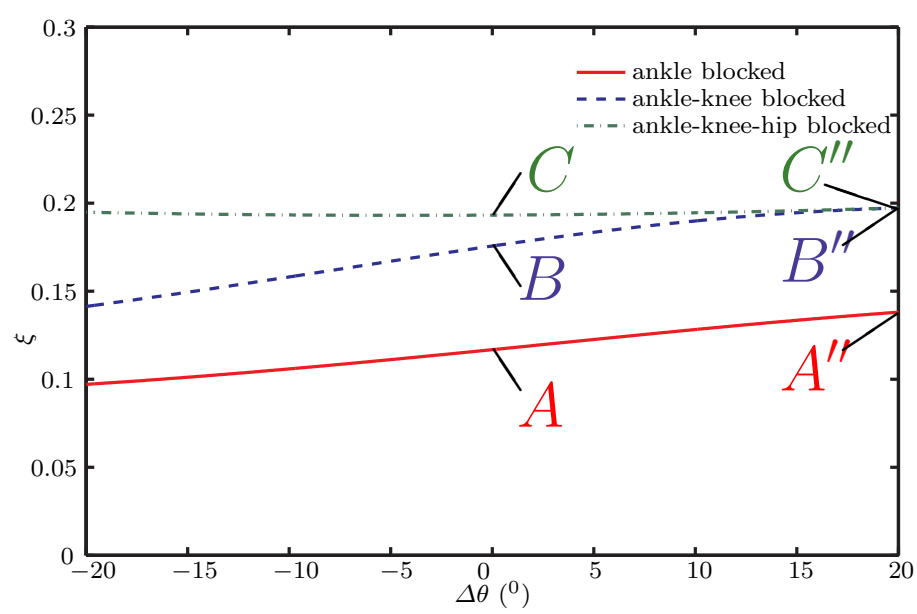

Fig. 6 Simulation Group II: Energetic performance index, $\xi$, vs, Torso-Pelvis angle deviation, $\Delta \theta$.

2. If in addition the leading knee is blocked, the energy dissipated can be greater than $19 \%$. In this situation, the torso angle can be used to modestly regulate the energy dissipated at the heel-strike down to a $14 \%$ for the range analyzed.

3. If in addition the leading hip is blocked, the dissipation is a $19 \%$, coincident with the maximum value for the previous situation. Most importantly, the torso angle does not have an appreciable effect on the dissipation.

These results are in agreement with the increase of energy loss presented in [17], in which a simpler, compass-like model was analyzed.

As before, the impulsive motion decomposition presented in Section 3 can be used to explain the effect of the Torso-Pelvis orientation change on the energetic performance index, $\xi$. Even though this change of configuration does not affect the pre-impact velocity, it does change the tangent space on the configuration manifold, and hence the redistribution of energy between the SCM an SAM. This explains the change found in the amount of dissipated energy. Note that, for the simulations in this section, all the energy associated with the SCM is dissipated.

Some of these aspects can be appreciated in Fig. 7, where the orthogonal decomposition of the dynamics at heel strike at three representative simulation points is illustrated: In simulation point $A^{\prime \prime}$ the leading ankle is blocked and the torso is at the maximum backward leaning position studied. In point $B^{\prime \prime}$ the knee is additionally blocked and in $C^{\prime \prime}$ the colliding leg hip is also blocked. In points $B^{\prime \prime}$ and $C^{\prime \prime}$ the torso configuration is as in $A^{\prime \prime}$. It is interesting to note that the initial velocity and the set of blocked joints are the same as for the situations $A$, $B$, and $C$ in Fig. 4 . Note that the differences between both simulation groups are therefore related only to the change of the SAM and SCM induced by the change of the configuration of the Torso-Pelvis segment.

As it has been reasoned in the previous section, although for a limited number of points, Fig. 7 along with Fig. 4 give an additional information that is helpful for understanding the heel strike event dynamics. As with the preceeding Simulation Group $I$, it is clear that the additional blocking of the knee alters not only the 
Fig. 7 Mass orthogonal decomposition of the movement for the points $A^{\prime \prime}, B^{\prime \prime}$, and $C^{\prime \prime}$. From left to right: total (SM), admissible (SAM) and constrained (SCM) pre-impact velocities of reference points.

magnitude of the different velocities in the SAM and SCM, but also their relative magnitudes. This relative change is not as significant when the leading hip is additionally blocked. This agrees with our previous conclusion that the additional blocking of the hip has the effect of reinforcing the dissipation introduced by the knee, without having relevant side effects on the distribution of velocities. As in the previous section, the blocking of the ankle alone produces an important after impact velocity of the leading knee center.

From the results of the last two sections, the leading ankle blocking reveals as the most important single contribution to the kinetic energy dissipation. Intuitively, it looks like a variable level of blocking can be used to change the amount of kinetic energy from a close to zero value, up to the high values related to the situation with complete blocking of the colliding leg ankle and colliding leg ankle and knee. This is the main subject of analysis in the next section.

\subsection{Simulation group $I I I$ : Effect of partial blocking of the leading ankle}

In this section, a variable post-impact ankle joint relative velocity is considered. We consider that during the impact muscles do not completely constrain the motion 
of the ankle, leading to a non-zero post-impact relative velocity for the joint. We call partial blocking to this non-complete blocking of the joint. It is expected that the impact gets stronger as the blocking of the leading ankle is more complete. That is, when the post-impact ankle joint relative velocity gets closer to zero.

Fig. 8 presents the plots of the energetic performance index, $\xi$, defined in Eq. (23), for different values of the post-impact ankle relative velocity, $\dot{\theta}_{A}^{R+}$. A variable blocking of the leading ankle has been used in all the simulations. Additionally, complete knee and hip blocking have also been simulated as it is indicated in the legend of the figure.

As in the previous section, to set up the initial condition in the simulations the system of equations (3) is solved using a constant value of $\omega_{0}=-1 \mathrm{rad} / \mathrm{s}$ for the trailing ankle joint velocity. That is, initial configuration and velocity is the same as for simulation points $A, B$, and $C$ defined in the previous sections. We limit the results to the values of $\dot{\theta}_{A}^{R+}$ for which $\xi>0$. To compare the results in this section with the simulations in previous ones, it is important to note that points $A, B$ and $C$, in Figs. 3, 4 and 8 represent the same simulation conditions.

As expected, as it can be seen from Eq. (24), the maximum energetic loss appears at $\dot{\theta}_{A}^{R+}=0$, when the colliding leg ankle is completely blocked. Also, as the pre-impact velocity is the same for all situations, Eq. (24) reveals that the plots shown should be parabolic with a maximum centered at $\dot{\theta}_{A}^{R+}=0$. This is in agreement with the results shown in Fig. 8.

The main conclusions obtained from this analysis are:

1. The partial blocking of the leading ankle alone can regulate from $\approx 0 \%$ up to $100 \%$ of the pre-impact total kinetic energy in the SCM, a $12 \%$ of the total pre-impact kinetic energy.

2. The additional blocking of the leading knee increases the pre-impact kinetic energy in the SCM to a $17 \%$ of the pre-impact total kinetic energy.

3. The additional blocking of the hip joint still produces a perceptible increase of the pre-impact kinetic energy in the SCM to a $19 \%$ of the pre-impact total kinetic energy.

These results put into perspective the great possibilities that the variable rigidization of the ankle joint can play on the heel strike dynamics. Partial blocking of the colliding leg ankle is able to completely regulate from close to $0 \%$ up to $100 \%$ the dissipation of all the dissipable energy: the pre-impact kinetic energy in the SCM. This amounts to a $12 \%, 17 \%$ or $19 \%$ of the total kinetic energy of the system depending on the blocking configuration.

Although detailed results are not presented, the same method has been used to analyze the total blocking on the trailing leg joints as well. Locking these joints does not significantly change the dissipation of energy with respect to that given in Fig. 8, when no joints in the swing leg are blocked. This result is in accordance with the passive role attributed to the swing leg in the heel strike dynamics that has been referred in the introduction [4]. This, in turn, supports the methods and conclusions proposed in this study.

The model in this study does not account for the contribution of motions out off the sagittal plane. For the present study, the major error contributions related to this simplification are probably those related to the lateral balancing and to the transverse rotation of the torso and pelvis. The small range of these motions contribute only for a small percentage of the kinetic energy in the SAM. 


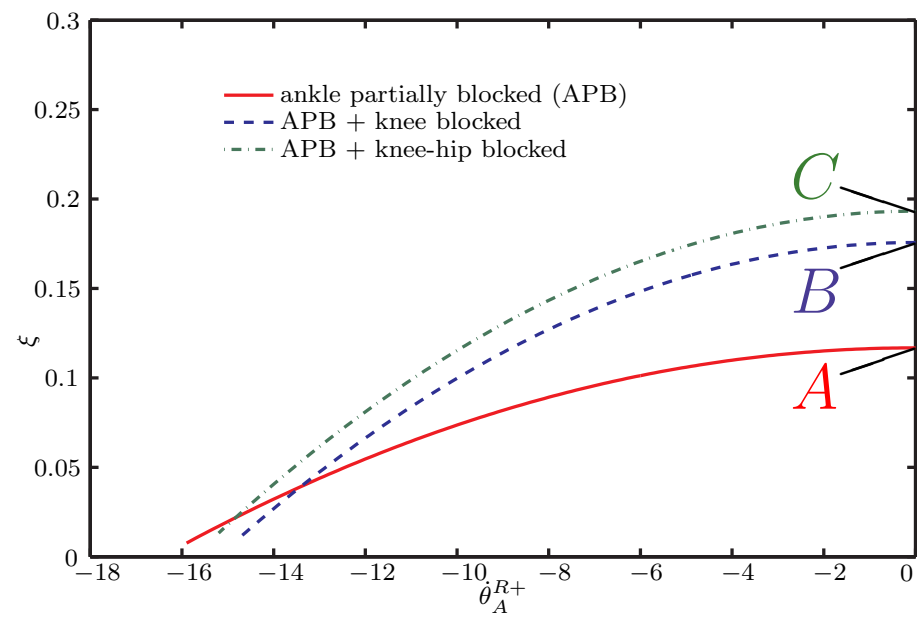

Fig. 8 Simulation Group $I I I$ : Energetic performance index, $\xi$, vs leading ankle angular velocity, $\dot{\theta}_{A}^{R+}$, for different blocking strategies.

The other neglected contribution is that of the movement of the arms relative to the torso, this is also small due to the relative small mass of the arms. However, as the arms are not blocked during the heel strike event, the part of the kinetic energy in the SCM is even smaller. Transversal rotation and balancing are barely constrained by the heel strike event: as the colliding heel is close to the sagittal plane the impulsive moment contribution to these non-sagittal portion of the SAM is small. This intuitive and non-formal discussion allows to see that, even with the simplifications related to the use of a $2 D$ model without arms, the most relevant contributions to the energetics of the heel strike dynamics presented in this article are qualitatively correct.

\section{Conclusions}

We have started this paper reminding the acknowledged role that the existing bibliography gives to mechanical energy dissipation at heel impact on bipedal locomotion stability. Based on this, we postulate that the heel strike can be accommodated in different ways to produce different levels of mechanical energy dissipation, and that this can be used as a regulating mechanism in the control of bipedal locomotion. Based on biomechanical principles, we also conclude that, within its physical limits, heel strike is by far the most efficient way to dissipate excess mechanical energy, and then to stabilize locomotion.

In this paper, we have built a seven-link $2 D$ realistic biomechanical model based on anthropometric data in order to do an impulsive dynamic analysis of the heel impact. Body configuration and velocity at the pre-impact instant are realistically defined and adjusted based on experimental data and observations. Three main control strategies are identified, namely, degree of ankle push-off, torso orientation, and partial blocking of the colliding ankle. Then, the proposed model and initial conditions are used in order to analyze the relevance of these controlling strategies. 
Accordingly three simulation groups are run, that in turn use different blocking strategies for the colliding leg joints. The impulsive dynamics formalism is used since it simplifies the required setup and gives a deeper insight into the dynamics of the impact. The computation and presentation of the results are based on the orthogonal decomposition of the dynamics at heel strike, as it allows for a deeper physical description and interpretation of the results.

The conducted study offers important conclusions about the role and relevance of the different controlling mechanisms studied during heel strike phase for normal gait. For instance, in relation with the trailing ankle push-off, based on the results obtained, we can conclude that increasing push-off is a very useful strategy to decrease energy loss at heel strike. In addition to the colliding ankle blocking, colliding knee and hip blocking allows to obtain different post-impact velocities for the same amount of dissipated energy. Knee blocking appears clearly as the mayor contributor to this velocity redistribution.

In relation with the Torso-Pelvis configuration, despite having some influence on the energetics of the impact, the amount of controlled energy is small enough to justify the absence of such a controlling mechanism during normal gait. Nevertheless, such mechanism can be useful in other situations, for example as an additional dissipation mechanism when the walker wants to stop.

It has been shown that with the partial blocking of the colliding ankle, a huge amount of energy (in the order of the total kinetic energy) can be continuously regulated, almost from zero up to a given maximum. This partial blocking can be combined with the blocking of the colliding knee and hip to alter the way in which motion redistributes after the impact. As before, knee blocking reveals as the mayor contributor in this respect. As it has been commented, the muscle actuation required to partially block a joint needs only a little amount of energy, conferring to the proposed mechanism even a bigger relevance.

\section{Acknowledgements}

Javier Ros gratefully acknowledges the support received from the Spanish Ministry of Education under the "Salvador de Madariaga" fellowship \#PR2009-0259 and from McGill University to enjoy a sabbatical research stay.

\section{References}

1. Mochon, S., McMahon, T.A., Ballistic walking, Journal of Biomechanics, Vol. 13, pp. 49-57, 1980.

2. Mochon, S., McMahon, T.A., Ballistic walking: An improved model, Mathematical Biosciences, Vol. 52, pp. 241-260, 1981.

3. Perry, J., Burnfield, J. M., Gait analysis: normal and pathological function, 2nd edition. Thorofare, New Jersey: SLACK Incorporated (2010).

4. Basmajian, J.V., The human bicycle, Biomechanics 5A (Komi, P.V., Ed.), University Park Press, Baltimore, MD, USA, 1976.

5. Alexander, R.M., Simple models of human motion, Applied Mechanics Reviews, Vol. 48, pp. 461-469, 1995. 
6. Hürmüzlü, Y., and Moskowitz, G. D., Bipedal locomotion stabilized by impact and switching: I and II, Dynamics and Stability of Systems, Vol. 2(2), pp. 73$112,1987$.

7. Hürmüzlü, Y., Moskowitz, G. D., The role of impact in the stability of bipedal locomotion, Int. J. Dynamical Systems, Vol. 1(3), pp. 217-234, 1986.

8. Goswami, A., Espiau, B., Keramane, A., Limit cycles and their stability in a passive bipedal gait, Proc. of the IEEE Conf. on Robot. and Automation, pp. 246-251, 1996.

9. Garcia, M., Chatterjee, A., Ruina, A., Coleman, M., The simplest walking model: stability, complexity and scaling, J. Biomechanical Engineering, Vol. 120(2), pp. 281-288, 1998.

10. Kuo, A.D., Donelan, J.M., Ruina, A., Energetic consequences of walking like an inverted pendulum: step-to-step transitions, Exercise and Sport Sciences Review, Vol. 33(2), pp. 88-97, 2005.

11. Kuo, A.D., Donelan, J.M., Dynamic Principles of Gait and Their Clinical Implications, Physical Therapy, Vol. 90(2), pp. 157-176, 2010.

12. Srinivasan, M., Ruina, A., Computer optimization of a minimal biped model discovers walking and running, Nature, Vol. 439(5), pp. 72-75, 2006.

13. Goswami, A., Thuilot, B., Espiau, B., A Study of the Passive Gait of a Compass-Like Biped Robot: Symmetry and Chaos, The International Journal of Robotics Research, Vol. 17(12), pp. 1282-1301, 1998.

14. McGeer, T., Passive dynamic walking, International Journal of Robotics Research, Vol. 9(2), pp. 62-82, 1990.

15. McGeer, T., Passive walking with knees, Proceedings of the IEEE Int. Conference on Robotics and Automation, Los Alamitos, CA, USA, pp. 1640-1645, 1990.

16. Donelan, J.M., Kram, R., Kuo, A.D., Simultaneous positive and negative external mechanical work in human walking, Journal of Biomechanics, Vol. 35, pp. 117-124, 2002.

17. Font-Llagunes, J. M., Kövecses, J., Efficient dynamic walking: Design strategies to reduce energetic losses of a compass walker at heel strike, Mechanics Based Design of Structures and Machines, Vol. 37(3), pp. 259-282, 2009.

18. Pain, M.T.G, Challis, J.H., Soft tissue motion during impacts: Their potential contributions to energy dissipation. Journal of Applied Biomechanics, Vol. 18, pp. 231-242, 2002.

19. Boyer, K.A., Nigg, B.M., Muscle activity in the leg is tuned in response to impact force characteristics, Journal of Biomechanics, Vol. 37(10), pp. 15831588, 2004.

20. Boyer, K.A., Nigg, B.M., Changes in Muscle Activity in Response to Different Impact Forces Affect Soft Tissue Compartment Mechanical Properties, Journal of Biomechanical Engineering, Vol. 129, pp. 594-602, 2007.

21. Lafortune, M.A., Hennig, E.M., Lake, M.J., Dominant role of interface over knee angle for cushioning impact loading and regulating initial leg stiffness, Journal of Biomechanics, Vol. 29 (12), pp. 1523-1529, 1996.

22. Murray, M.P., Drought, A.B., Kory, R. C., Walking Patterns of Normal Men, J. Bone Joint Surg Am, Vol. 46, pp. 335-360, 1964.

23. Pars, L.A., A Treatise on Analytical Dynamics, Heinemann, London, England, 1965. 
24. Pfeiffer, F. and Glocker, C., Multibody Dynamics with Unilateral Contacts, Wiley. 1996.

25. Font-Llagunes, J. M., Kövecses, J., Pàmies-Vilà, R., Barjau, A., Comparison of Impulsive and Compliant Contact Models for Impact Analysis in Biomechanical Multibody Systems, In Proceedings, $1^{\text {st }}$ Joint International Conference on Multibody System Dynamics, , Lappeenranta, Finland, 2010..

26. Font-Llagunes, Barjau A., Pàmies-Vilà R., Kövecses, J., Dynamic analysis of impact in swing-through crutch gait using impulsive and continuous contact models, Multibody System Dynamics, Vol. 28 (3), pp. 257-282, 2012.

27. Dumas, R., Chèze, L., Verriest, J.-P., Adjustments to McConville et al. and Young et al. body segment inertial parameters, Journal of Biomechanics, Vol. 40(3), pp. 543-553, 2007.

28. Ros, J., Gil, J., Zabalza, I., 3D_Mec. An Application to Teach Mechanics, In Proceedings of ASME, IDETC/CIE2005, Long Beach (California), U.S.A., 2005.

29. Kövecses, J., Dynamics of Mechanical Systems and the Generalized Free-Body Diagram-Part I: General Formulation, ASME J. Applied Mechanics, Vol. 75(6), 061012, pp. 1-12, 2008.

30. Kövecses, J., Dynamics of Mechanical Systems and the Generalized Free-Body Diagram-Part II: Imposition of Constraints, ASME J. Applied Mechanics, Vol. 75(6), 061013, pp. 1-8, 2008.

31. Font-Llagunes, J. M., Kövecses, J., Dynamics and energetics of a class of bipedal walking systems, Mechanism and Machine Theory Vol, Vol. 44(11), pp. 1999-2019, 2009.

32. Modarres Najafabadi, S.A., Dynamics modelling and analysis of impact in multibody systems, PhD Thesis, Department of Mechanical Engineering, McGill University, Montreal QC, Canada, 2008.

33. Kuo. A.D. Energetics of actively powered locomotion using the simplest walking model. ASME Journal of Biomechanical Engineering, 124(2):113-120, 2002. 